\title{
A Genetic Algorithm for Design of Moment-Resisting Steel Frames
}

\author{
Prakash Kripakaran \\ University of Exeter, \\ Exeter EX4 4QF, United Kingdom \\ Brian Hall, Abhinav Gupta \\ North Carolina State University, \\ Campus Box 7908, Raleigh NC 27695-7908
}

\begin{abstract}
This paper presents computational approaches that can be implemented in a decision support system for the design of moment-resisting steel frames. Trade-off studies are performed using genetic algorithms to evaluate the savings due to the inclusion of the cost of connections in the optimization model. Since the labor costs and material costs vary according to the geographical location and time of construction, the trade-off curves are computed for several values of the ratio between the cost of rigid connection and the cost of steel. A real-life 5-bay 5-story frame is used for illustration. Results indicate that the total cost of the frame is minimal when rigid connections are present only at certain locations. Finally, "Modeling to Generate Alternatives - MGA," is proposed to generate good design alternatives as the solution from optimization may not be optimal with respect to the unmodeled objectives and constraints. It provides a set of alternatives that are near-optimal with respect to the modelled objectives and that are also farther from each other in the decision space. Results show that a final design could be chosen from the set of alternatives or obtained by tinkering one of the alternatives.
\end{abstract}

\section{Introduction}

The design of moment-resisting steel frames is one particular class of problems that can significantly benefit from novel approaches for decision support. While the use of rigid connections in the frame can considerably reduce the moments in the beams and save significant material costs, the cost of fabrication of a rigid connection is often very high due to excessive labor requirements for on-site welding. Depending on the relative cost of a connection to the cost of material, an optimal solution is likely to have rigid connections at only certain locations within a frame. In the current practice, all connections within a rigid frame are assumed to be rigid. Even if an attempt is made to reduce the number of connections within the frame, all connections within certain bays of a frame are assumed to be rigid. The bays with rigid connections in the final design are identified by a hands-on trial that is based on professional judgment without any formal consideration of the connection cost. Such frames are not likely to be optimal when connection cost is included.

Research in steel frame optimization has generally focused on minimizing the total weight of steel. Both mathematical programming techniques (Simoes, 1996) and heuristic optimization techniques (Balling, 1991; Foley and Schinler, 2003; Kicinger et al, 2005; Xu and Grierson, 2006; Fragiadakis et al, 2006; Kargahi et al, 2006; Chan and Wong, 2007; Kameshki and Saka, 2007; Degertekin, 2008) have been used. Saka (2007) provides a summary of the evolutionary optimization techniques applied to frame weight optimization. However, cost models used in practice have changed over recent years. Labor costs have gradually risen such that they constitute $60 \%$ of the total construction cost (Liu et al, 2006) today. A state-of-the-art publication by the American Society of Civil Engineers (ASCE) on optimal structural design (Burns, 2002) recommends optimization formulations to consider the cost of fabrication and erection. In general, moment connections are 
modeled as either fully-rigid or hinged for analysis purposes. Some studies (Dhillon and O'Malley III, 1999; Foley and Vinnakota, 1999a,b) have found that modeling the semi-rigid behavior of connections can lead to lighter structures. Most of the rigid frame optimization studies, even those that model the semi-rigid behavior of connections (Machaly, 1986; Al-Salloum and Almusallam, 1995; Kameshki and Saka, 2001), focus on minimizing the total weight of the structure and do not incorporate the fabrication cost of the connection in their optimization model. Only a few frame optimization studies have incorporated both the connection cost and the cost of material. Simoes (1996) developed a linear programming approach for cost optimization whereas Xu and Grierson (1993) formulated an optimal semi-rigid frame design problem in which the cost of connections was represented by an equivalent weight of steel. Hayalioglu and Degertekin $(2004,2005)$ used genetic algorithms for optimization with this cost model. However, previous studies have not examined the cost savings in selecting the optimal connection types for each beam-column connection.

While minimizing the total cost is a primary objective of design optimization, often other objectives like redundancy are given consideration during the decision-making process. Since the number of rigid connections in a frame is directly related to its redundancy, a natural engineering judgment is to select designs that have a higher number of rigid connections. However, this may lead to a higher total cost since the cost of rigid connections are much larger than the cost of hinged connections. Therefore trade-off curves between these competing objectives can provide valuable insight into the problem. In addition to these quantifiable objectives like redundancy and cost, design problems often involve other objectives or constraints that are excluded from the optimization model because of their unquantifiable nature (Brill Jr. et al, 1990; Loughlin et al, 1995). As a result, the final solution is rarely the final design adopted by a decision-maker. An example of such an unquantifiable parameter can be an additional cost related to erecting a frame in a congested urban setting (Kripakaran and Gupta, 2006). This cost can be difficult to quantify but is often a critical factor in determining the design chosen for implementation. Therefore, it would be preferable to first evaluate a set of alternatives that is good with respect to the modeled objectives and constraints. Then, an alternative that is also good with respect to the unmodeled objectives and constraints can be selected based on professional judgment. Computational approaches that use optimization to not only find the "best" solution, but also generate a set of "good" alternatives permit the incorporation of professional expertise for arriving at a final design and for conducting investigative studies like trade-off and what-if analyses.

In this paper, we propose computational approaches that can become part of a decision support system for the design of moment-resisting frames. The study uses a cost function that considers the material cost of steel as well as the cost of rigid connections in the frame. For simplicity, connections are considered either fully-rigid or hinged. Semi-rigid behavior is not considered in this exploratory study. Moreover, in practice, engineers continue to model connections as fully rigid or hinged due to the complexity involved in modeling semi-rigid behavior. Unlike many optimization formulations in which the beam and column member types are the only decision variables, the proposed formulation uses binary decision variables that correspond to the presence or absence of a rigid connection at each beam-column junction. We propose a Genetic Algorithm (GA), that uses a unique crossover scheme, for finding the minimum cost design since the optimization formulation involves discrete decision variables and a search space that is combinatorial. Previous studies (Rajeev and Krishnamoorthy, 1997; Gupta et al, 2005) have shown that GAs can provide superior performance for discrete structural optimization problems. Even though GAs (Eiben and Smith, 2003; Haupt and Haupt, 2004) can be computationally-expensive, if correctly tuned they are efficient global search methods that have the ability to consistently provide a near-optimal solution in highly multi-modal search spaces. In the proposed GA, the use of penalty functions for stress and displacement constraints is avoided by developing novel algorithms to change infeasible solutions into feasible solutions. Trade-off studies between the number of rigid connections and the total cost are performed using the GA with this unique crossover scheme. "Modeling to Generate Alternatives - MGA," which is an optimization-based technique, is used to identify alternatives that are far apart in decision space but close to the optimal solution in objective space. The solutions from the MGA are examined with respect to various design aspects that are not formally considered in the optimization model. 


\section{Problem definition}

A mathematical model of the frame optimization problem is presented in this section. Consider a $m$-story, $n$-bay plane frame. It consists of $(m \times n)$ beams. An example frame for $m=5$ and $n=5$ is shown in Figure 1. Each beam in the frame has two connections, one at each junction with the columns. Let us number the possible rigid connection locations from 1 to $R$ where $R=2(m \times n)$ is the total number of rigid beam-column connection locations in the frame. Let $c_{i}$ represent the decision variable corresponding to the presence or absence of a rigid connection at location $i, i=1, \ldots, R$. The presence of a hinged connection at location $i$ is represented by $c_{i}=0$ and the presence of a rigid connection by $c_{i}=1$. Then, the binary string $<c_{1}, c_{2}, c_{3}, c_{4}, \ldots, c_{R}>$ represents the decision variables that correspond to the various beam-column connections in the frame. In many cases, the number of connection decision variables may be less than $2(m \times n)$ because of considerations of symmetry in the frame. In such a case, $R$ would represent the total number of connection locations after considering symmetry. The other set of decision variables in the problem is related to the product types $p_{j}$ for the various members in the frame. Let $<p_{1}, p_{2}, \ldots, p_{2 m n+m}>$ represent the decision variables that correspond to the product type for each of the $(2 m n+m)$ members in the frame. The total cost $C$ of the frame is expressed as,

$$
\text { Total cost, } C=T_{s}+T_{c}
$$

in which $T_{s}$ and $T_{c}$ correspond to the cost of steel and connections, respectively. $T_{s}$ is computed as,

$$
T_{s}=C_{s} \sum_{j=1}^{2 m n+m} w_{j}\left(p_{j}\right) l_{j}
$$

where $w_{j}$ is the weight in metric tons per unit length of the product $p_{j}$, which is the product (or member type) assigned to member $j . l_{j}$ is the length of member $j . C_{s}$ is the cost per metric ton of steel. The total cost of the connections, $T_{c}$, is computed as,

$$
T_{c}=C_{r} \sum_{i=1}^{R} c_{i}
$$

$C_{r}$ is the cost of a single rigid connection. The cost of hinged beam-column connections is not included in this model as it is significantly less than the cost of rigid beam-column connections.

The material cost of steel $C_{s}$ varies over time. The cost of a rigid connection $C_{r}$ is heavily dependent on the local labor costs. This study was conducted in the state of North Carolina where $C_{s}$ and $C_{r}$ are approximately equal to $\$ 600$ and $\$ 900$ respectively. Performing the optimization study for only these values may mean that the scope of this research is restricted to this region. For the sake of generality optimization studies are conducted for various values of the ratio between $C_{r}$ and $C_{s}$ by modifying Equation 1 as follows.

$$
\begin{gathered}
\text { Total cost, } C=C_{s} F \\
F=\sum_{j=1}^{2 m n+m} w_{j}\left(p_{j}\right) l_{j}+\frac{C_{r}}{C_{s}} \sum_{i=1}^{R} c_{i}
\end{gathered}
$$

$F$ can be considered as the total equivalent weight of steel and includes the contribution from both members and connections. The generated trade-off curves are plotted between the number of rigid connections $r_{r e q}$ and $F$ for different values of $C_{r} / C_{s}$. In this study, trade-off curves are obtained for values of $C_{r} / C_{s}$ between 1.0 and 2.0. $C_{r} / C_{s}$ is equal to 1.5 in the state of North Carolina.

The constraints for the design problem are prescribed by the strength and serviceability requirements. The strength requirements are specified in the Manual of Steel Construction, Load and Resistance Factor Design (AISC, 2001). According to the manual, the members in the frame must conform to the following design equations for each load case.

For $P_{u} / \phi P_{n} \geq 0.2$,

$$
\frac{P_{u}}{\phi P_{n}}+\frac{8}{9} \frac{M_{u}}{\phi_{b} M_{n}} \leq 1.0
$$


For $P_{u} / \phi P_{n} \leq 0.2$,

$$
\frac{P_{u}}{2 \phi P_{n}}+\frac{M_{u}}{\phi_{b} M_{n}} \leq 1.0
$$

$P_{u}$ is the factored axial load (tensile or compressive) in the member, $P_{n}$ is the nominal (tensile or compressive) strength of the member and $\phi$ the corresponding resistance factor. For tension, $\phi=0.75$ in Equation 6 and $\phi=0.9$ in Equation 7. For compression, $\phi=0.85$ in both equations. $M_{u}$ and $M_{n}$ are the required and nominal flexural strength for major axis bending, respectively. $\phi_{b}$ is the resistance factor for flexure and is equal to 0.9 . Since the proposed study considers only plane frames, Equations 6 and 7 have only those terms that correspond to major axis bending. $P_{n}$ is calculated using the area of the member and either the yield stress or the buckling stress depending upon the nature of the axial force in the member, i.e., tension or compression, respectively. $M_{n}$ is calculated using certain equations that evaluate the ability of the member to resist the following buckling possibilities - local flange buckling, local web buckling and lateral torsional buckling. These equations are described in detail in AISC (2001) and are not provided in this paper for brevity. The serviceability constraint given below governs the maximum side sway $\delta$ for any story of height $H$ in the frame.

$$
\delta \leq \frac{H}{400}
$$

For a given story, the sway is evaluated as the maximum difference in the horizontal displacements between the two floor slabs that bound the story.

\section{Optimization approach}

The goal of optimization is to find values for $c_{i}$ and $p_{j}$ that minimize the total cost $C$. In a conventional genetic algorithm (Goldberg, 1989; Michalewicz and Fogel, 2004), all variables, $c_{i}$ as well as $p_{j}$, are modeled into the GA representation. The constraints are normally applied by having penalty costs in the objective function of the GA. A key limitation of such an approach is the excessively large search space that arises due to the large number of decision variables and the numerous types of products available for the members in the frame. This large search space diminishes the efficiency as well as the quality of the search process. However, a closer inspection of this particular problem reveals a dependency between the variables $c_{i}$ and $p_{j}$. For a given set of values for $c_{i}$, there exist certain products corresponding to $p_{j}$ that will minimize the total cost of the frame. Therefore, it is not necessary to consider $p_{j}$ as the decision variable in the GA representation. The optimization formulation needs to model only $c_{i}$ as decision variables. This modification can significantly reduce the size of the search space. This approach is essentially an example of problem decomposition in which a GA minimizes the total cost by changing connection types and locations and the total cost for each solution within the GA is evaluated by finding the minimum weight solution for a specified connection configuration.

The proposed optimization approach is therefore focused on finding the optimal values of $c_{i}$ and hence, the best locations for rigid connections in a frame. The proposed approach involves two key steps. In the first step, we determine a minimum weight frame using the algorithm described in Section 4. To do so, we assume that all connections in the frame are rigid and find the products for the various members that minimize the total weight of the frame. In the second step, we use a GA (see Section 5) to perform a trade-off study between the number of rigid connections and total cost. This trade-off study is performed by conducting a series of GA runs, where each GA run is aimed at finding the solution with minimal total cost for a specified number $r_{r e q}$ of rigid connections. During a GA run for a specified $r_{r e q}$, the GA explores the solution space with exactly $r_{r e q}$ rigid connections. The fitness of each solution that is generated within the GA is evaluated using the algorithm given in Section 5.1. This algorithm starts with a frame having a connection configuration as defined by the solution, and the products as evaluated for the minimum weight solution in the previous step. Since this frame has rigid connections at only certain locations, the products evaluated for the minimum-weight frame may not be sufficient to meet the sway and/or Load and Resistance Factor Design (LRFD) requirements. Therefore the products for the frame are modified using algorithms (see Section 5.1) that change the member sizes (product types) to find the minimal-cost feasible solution for that connection configuration. If the solution is not repairable, penalties are assigned to force the GA toward 
feasible settings of connections. The various components of the proposed approach are described in detail in the following sections.

\section{Algorithm for least-weight frame}

As stated earlier, we first find the minimum weight solution when all the connections in the frame are rigid connections, i.e, $\left\{c_{i}=1, \forall i\right\}$. This solution is generated by following the simple iterative procedure outlined below. This algorithm is similar to that for finding a fully-stressed design (Patnaik and Hopkins, 1998). It attempts to find the least weight solution that also satisfies strength and sway requirements. While the algorithm presented here is not guaranteed to find the true least-weight solution, its purpose is to evaluate an initial set of product types for the members in the frame that can later facilitate finding $p_{j}$ within the GA implementation.

Step 1: Model the frame with only rigid connections.

Step 2: Assign the smallest W-shape member to all the beams and columns.

Step 3: Analyze the structure considering P- $\delta$ effects for the various load combinations and evaluate the acceptability with respect to the various strength design requirements using Equations 6 and 7.

Step 4: Use algorithm given in Figure 2 to find a solution that satisfies strength design requirements.

Step 5: Check if the sway requirements are met for each floor.

Step 6: Use algorithm given in Figure 3 to find a solution that satisfies sway requirements. The algorithm assigns $\mathrm{W}$-shapes with a larger value for area moment of inertia to all beams and columns in a floor that violate sway requirements such that the increase in area moment of inertia $\left(\Delta I_{\text {req }}\right)$ for each member satisfies the following equality.

$$
\Delta I_{r e q}=\frac{\left(\delta-\delta_{u}\right)}{\delta} I\left(p_{j}\right)
$$

$\delta$ is the floor sway obtained from analysis and $\delta_{u}$ is the maximum allowable floor sway. $I\left(p_{j}\right)$ is the moment of inertia of product $p_{j}$ that is currently assigned to member $j$. The premise for using Equation 9 is based on the direct relationship of the displacement to bending stiffness of beams and columns.

The above algorithm is not computationally intensive and converges to a feasible solution after only a few iterations over both the strength and the sway constraints. The performance of the heuristics is illustrated in Section 7. Additional constraints may also be considered. For example, the W-shape of a column member in any floor must be larger than that of any column member above it. Such rules can also be appropriately implemented in the above algorithm while performing steps 4 and 7 . The set of products evaluated using the algorithm described in this section is used in the algorithms of the GA implementation, which is described in the following section.

\section{GA implementation}

The GA approach presented in this paper is developed to generate the trade-off curve between the number of rigid connections, $r_{r e q}$, and the total cost, $T_{c}$. As mentioned previously, this curve is generated using a series of GA runs, where each GA run is aimed at minimizing the total $\operatorname{cost} C$ for a specified $r_{r e q}$. The variables $c_{i}$ are the only decision variables that are affected by the GA operators. We use a binary string representation in the GA that is given as $\left\langle c_{1}, c_{2}, \ldots, c_{R}\right\rangle$. The variables for the product types $p_{j}$ are implicitly related to those for connection types. For a particular configuration of rigid and hinged connections, there is only one set of values for the product variables such that the total cost is minimal. Therefore, during fitness evaluation in the GA, each connection configuration is evaluated by finding the values for the product variables that reduce total cost. Using a computationally-intensive approach such as a GA to evaluate the optimal values for product variables can significantly increase the time required to evaluate the fitness of a single combination of connections. Therefore the optimal values for the product variables are found using the following heuristic algorithm. 


\subsection{Fitness evaluation}

Given a particular binary string, the GA evaluates its fitness in the following manner.

Step 1: Consider a frame with the products, as determined using the algorithm in Section 4, assigned to its various members and having the connection configuration represented by the binary string.

Step 2: Determine if the frame satisfies strength constraints. If not, use the algorithm given in Figure 2 to transform this solution into one that satisfies strength constraints.

Step 3: Determine if the frame satisfies floor sway constraints. If not, use the algorithm given in Figure 3 to transform the solution into one that satisfies floor sway constraints.

Step 4: Evaluate the fitness of the solution using the following fitness function,

$$
Z=-\left(C+P_{s}+P_{d}\right)
$$

in which $C$ is the total cost of the frame whereas $P_{s}$ and $P_{d}$ are the penalty costs that correspond to the various constraint violations, i.e., strength and sway, respectively. $P_{s}$ and $P_{d}$ are applied only in the event that the algorithms used in steps 2 and 3 are unable to force the solution to satisfy the respective constraints. These penalty costs are calculated as follows.

$$
\begin{gathered}
\qquad P_{s}=a \sum_{j=0}^{2 m n+m} s_{j} \\
s_{j}=\left\{\begin{array}{l}
0, \text { if the corresponding design equation is satisfied } \\
\text { Left-hand side of design equation, if member violates design equation }
\end{array}\right.
\end{gathered}
$$

The design equations that are referred to above are Equations 6 and 7. $a$ is a constant multiplier and is set equal to 100,000. A large value is chosen for $a$ to give a high cost to solutions that do not satisfy strength constraints even after applying the algorithm in Figure 2. Penalty cost $P_{d}$ is evaluated as,

$$
\begin{gathered}
P_{d}=b \sum_{j=0}^{2 m n+m} d_{j} \\
d_{j}=\left\{\begin{array}{l}
0, \text { if member } j \text { is a beam or } \delta_{j}-\delta_{u} \leq 0 \\
\delta_{j}-\delta_{u}, \text { if member } j \text { is a column and } \delta_{j}-\delta_{u} \geq 0
\end{array}\right.
\end{gathered}
$$

$\delta_{j}$ is the floor sway for column $j$ and is simply the absolute difference of the horizontal displacements at the two end nodes of the member. $\delta_{u}$ is the maximum allowable sway as specified by Equation $8 . b$ is a constant multiplier and is equal to 100,000. As for $a$ in Equation 11, $b$ is also given a large value to assign a high cost to solutions that remain infeasible with respect to serviceability constraints even after applying the algorithm given in Figure 3.

\subsection{Initialization of the GA}

Since a particular GA run must consider only those solutions that have a specified number of rigid connections, $r_{r e q}$, strings in the GA must satisfy the following property, $\sum c_{i}=c_{r e q}$. An additional variable $c_{r e q}$ is used since symmetries of the frame may be imposed in the string representation and consequently $c_{r e q}$ may be less than $r_{r e q}$. Initializing the GA appropriately can minimize the number of solutions on which this penalty is used. The following seeding technique is used to ensure that all solutions in the initial population have exactly the specified $r_{r e q}$ number of rigid connections.

$$
c_{i}=\left\{\begin{array}{l}
1, \text { for } i \in S \\
0, \text { for } i \notin S
\end{array}\right.
$$

$S$ is a set of cardinality $c_{r e q}$ with elements randomly chosen from the collection $\{1,2,3, \ldots, R\}$ such that no two elements in $S$ are equal. 


\subsection{GA operators}

In an attempt to improve the quality of solutions in a subsequent generation, the GA uses the crossover operator to produce two offsprings by combining two parent strings. Traditional GA approaches use uniform crossover or one-point crossover. These conventional crossover operators can generate solutions in which the number of rigid connections is different from $r_{r e q}$. Such a situation is avoided by using the crossover operator proposed by Gupta et al (2005). This crossover operator has the unique characteristic of generating offsprings that have an equal number of ones when each of the parent strings also have the same equal number of ones. This crossover is illustrated in Figure 4. Let us consider two parent strings, which have exactly $c_{r e q}$ number of ones, given by $\left\langle c_{a 1}, c_{a 2}, \ldots, c_{a R}>\right.$ and $\left\langle c_{b 1}, c_{b 2}, \ldots, c_{b R}>\right.$. Then, define the set $S=\left\{i: c_{a i} \neq c_{b i}\right\}$. Note that $S$, whose cardinality is always even, can be randomly divided into two sets of equal sizes $S_{1}$ and $S_{2}$. The offsprings, $\left\langle c_{c 1}, c_{c 2}, \ldots, c_{c R}>\right.$ and $\left\langle c_{d 1}, c_{d 2}, \ldots, c_{d R}>\right.$, are then given by:

$$
\begin{gathered}
c_{c i}=\left\{\begin{array}{c}
1, \forall i \in S_{1} \\
0, \forall i \in S_{2} \\
c_{a i}, \forall i \notin S
\end{array}\right. \\
c_{d i}=\left\{\begin{array}{l}
1, \forall i \in S_{2} \\
0, \forall i \in S_{1} \\
c_{a i}, \forall i \notin S
\end{array}\right.
\end{gathered}
$$

This crossover scheme effectively reduces the size of the search space, which could be as high as $2^{R}$ when using uniform crossover, to the combinatorial expression $C_{c_{r e q}}^{R}=\frac{R !}{c_{r e q} !\left(R-c_{r e q}\right) !}$. A crossover probability of 0.75 is used for the study.

GAs use the mutation operator to make random changes to existing solutions in a population. The premise for mutation is that random changes may result in solutions that lie in those regions of the decision space that were not explored in the previous generations. A mutation probability of $0.1 \%$ per bit is chosen according to accepted practice (Mitchell, 1998) within the GA community. For the trade-off study, the decision space consists of all solutions that have exactly $r_{r e q}$ number of rigid connections, i.e., $c_{r e q}$ number of ones. Therefore, we use the following mutation operator, which makes random changes but does not alter the number of ones in the string. Let $\left\langle c_{1}, c_{2}, \ldots, c_{R}>\right.$ represent the solution. Then, $S_{1}=\left\{i: c_{i}=1\right\}$ and $S_{2}=\left\{i: c_{i}=0\right\}$ represent the locations that have rigid connections and hinged connections respectively in the original solution. For mutation, set $c_{k}=0$, where $k$ is randomly chosen from $S_{1}$ and set $c_{l}=1$, where $l$ is randomly chosen from $S_{2}$.

Binary tournament selection is employed in the GA. The probability that the better solution is chosen from a tournament is 0.75 . The convergence criterion is determined based on the number of consecutive generations for which there is no change in the global optimal solution. If this number exceeds 10, then the GA is considered to have converged to the optimal solution.

\section{Plane frame example - description}

The performance of the proposed optimization approach is studied for a real-life 5-story 5-bay frame. This frame is one of two end frames in a commercial building and is used primarily to resist the lateral forces arising due to wind in one direction. The elevation view of the frame is shown in Figure 1.The figure shows the numbering of the members and the joints in the frame. Each story has a height of $11.5 \mathrm{ft}(3.5 \mathrm{~m})$. Each bay is $20 \mathrm{ft}(6.1 \mathrm{~m})$ wide except for the central bay which has a width of $36.5 \mathrm{ft}(11.1 \mathrm{~m})$.

Load factors and load combinations for design are taken from the $3^{\text {rd }}$ edition of AISC Manual of Steel Construction for Load and Resistance Factor Design (AISC, 2001). The strength of moment frame is evaluated for the following load combinations $-1.4 D,\left(1.2 D+1.6 L+0.5 L_{r}\right)$ and $\left(1.2 D+1.6 W+0.5 L+0.5 L_{r}\right)$, where $D, L, W$ and $L_{r}$ represent dead loads, live loads, wind loads and roof live loads respectively. Note that there are effectively four load combinations as the wind load results in two different load cases that correspond to the wind blowing in either direction. The details of all the loads are summarized in Tables 1 and 2. 
The serviceability of the building is evaluated for two load cases corresponding to only the wind loads acting on either side of the frame.

The W-shapes for the members of the frame are chosen from the shapes listed in the AISC Manual of Steel Construction for LRFD (AISC, 2001). Engineers follow certain guidelines for assigning products to different frame members. The following guidelines are used in this study.

- The AISC manual has a table of W-shapes that have the highest area moment of inertia for lowest weight per unit length among groups of $\mathrm{W}$-shapes with values of similar order of magnitude for area moment of inertia. These W-shapes that have the largest area moment of inertia for minimal weight are best for flexural design. Beams are assigned products from a separate product set with only these W-shapes.

- All members that belong to a particular column in the frame use W-shapes that have the same web depth. Engineers advise using W14 shapes for columns and hence, a separate product set, which is composed of only W14 shapes for columns, is used.

These two product sets are given in Tables 3 and 4.

The frame has a total of 25 beams and each beam has junctions with columns at its two ends. Therefore there are 50 beam-column junctions and consequently, 50 possible locations for rigid connections. Due to the symmetry in both the loading and the geometry about axis A-A in Figure 1, only 25 decision variables $-\left\langle c_{1}, c_{2}, \ldots, c_{25}\right\rangle$ are needed in the optimization formulation. While the frame has 55 members, only 30 decision variables for the products $-<p_{1}, p_{2}, \ldots, p_{30}>$, are needed due to symmetry.

\section{Results}

As a first step, we use the algorithm to find the least weight solution when the frame consists of only the rigid connections, i.e., all 50 connections are considered to be rigid. The $\mathrm{W}$-shapes $p_{j}$ assigned to the various members in the solution from this step are provided in Table 5. During this process, it is observed that the floor sway constraints govern the search for this solution. Therefore, this solution is likely to violate the sway constraints if the frame is assigned a fewer number of rigid connections. The weight of steel in this solution is approximately 20 metric tons. Assuming that the frame is considered for construction in the state of North Carolina, the ratio of costs is $C_{r} / C_{s}=1.5$. Since all 50 connections are rigid, the total steel equivalent weight of the frame $F$ can be calculated from Equation 5 as $F=50 \times 1.5+20=95$ metric tons.

In order to illustrate the performance of the heuristics used in this algorithm, results are compared to those obtained using a simple GA for member sizing. An integer representation and uniform crossover are employed in the GA. A mutation operator that alters the product assigned to a randomly chosen member of the frame is specified. A mutation probability of $0.1 \%$ per bit is chosen. All connections are assumed to be rigid. The population size is 400 . The best solution produced by the GA over 5 runs has an equivalent weight of 99 metric tons. While the GA performs over 19600 evaluations on average, the algorithm given in Section 4 requires fewer than 40 evaluations. The inferior performance of the GA can be attributed to a combinatorial search space due to 30 decision variables (number of frame members assuming symmetry) and 37 possible values for each variable (number of product types available for each member). These results show that the heuristics are computationally efficient and also produce a better solution compared to the GA.

In the second step, we generate the trade-off curve using the GA described in Section 5. A population size of 200 is specified in the GA. The trade-off curve is generated for $r_{r e q}=4, \ldots, 36$. Due to symmetry, $c_{r e q}=r_{r e q} / 2$. The resulting trade-off curve is shown in Figure 5. The solutions plotted on the curve are the best solutions generated over a series of five GA runs each with a different random starting population. The trade-off curve clearly shows that there is a certain number of rigid connections, $r_{o p t}$, for which the total cost is optimal. As $\left|r_{r e q}-r_{\text {opt }}\right|$ increases, the total cost of the solution also increases. The robustness of the GA has also been verified and these results are also shown in Figure 5 using box plots that illustrate the range of values obtained for total cost from five different runs for various values of $r_{r e q}$. The figure shows that the GA consistently generates a good solution for several values of $r_{r e q}$ for the 5-bay 5-story frame with a fully fixed base. 
Another relation that is of interest is the variation of material cost with number of rigid connections $\left(r_{r e q}\right)$. This is plotted in Figure 6. This is obtained by subtracting the cost of rigid connections from the total cost in Figure 5. As expected, the total material cost of the frame is observed to decrease with increasing numbers of rigid connections. The plot also shows that the savings in material cost obtained by adding a rigid connection reduces as $r_{r e q}$ increases. This could be used to determine a upper limit for $r_{r e q}$ beyond which there is no benefit in continuing to generate further points on the trade-off curve.

The trade-off curves provide an insight into the relationship between total cost and number of rigid connections in a frame. Specifically, it gives engineers an estimate of the number of rigid connections required for minimal total cost. It also gives an idea of the additional cost that may be incurred upon including a rigid connection in a given solution. Engineers may employ the solutions on the trade-off curves for design. They may also use them to generate further alternatives as described in Section 8. From Figure 5, $r_{o p t}=10$. $F=46.00$ metric tons for this solution. The rigid connection locations for this solution are indicated by the empty circles in Figure 7. The other beam-column connections are hinged connections. The W-shapes assigned to the various members are also shown in Figure 7.

Often, high costs associated with footings may force the designers to consider the connections at the base of the frame as hinged. The trade-off study is repeated for such a case. Instead of using the algorithm for finding the least-weight frame, values for $p_{j}$ from the previous case, which are given in Table 5, are used. This procedure is acceptable since the idea is to begin with an initial set of products that can later be modified within the GA. As the set of products given in Table 5 are obtained for a fixed base, using the same products for a hinged base would lead to frames that would have to be strengthened to withstand higher bending moments. This can be effectively performed within the GA by the algorithm given in Figure 2. The results of this trade-off study are plotted in Figure 8. In this case, the total cost appears to, in general, increase with increasing numbers of rigid connections. It is observed that there are no feasible solutions for $r_{r e q}<6$. Solutions for $r_{r e q}>20$ are not generated as the cost will only increase monotonically with increasing number of rigid connections. Moreover, as shown in Figure 6, the reduction in material cost achieved by adding a rigid connection tapers after $r_{r e q}=20$. The cost optimal solution is for $r_{r e q}=6$ and its details are given in Figure 9. The robustness of the GA is also verified for this case and the corresponding boxplots are shown in Figure 8. As shown in the figure, a higher degree of variability in results is observed for the frame with a fully hinged base.

Since the values for $C_{r} / C_{s}$ are expected to change with time and location, we examine the sensitivity of the study for different specified values of $C_{r} / C_{s}$. We perform the study only for the case when all the supports in the frame are fixed. The effect of the number of rigid connections $r_{r e q}$ on the total equivalent cost of steel is recalculated for five different values of $C_{r} / C_{s}$ between 1.0 and 2.0. This does not require separate optimization runs for each value for $C_{r} / C_{s}$ since the ratio affects only the evaluation of the cost. The optimal connection configurations and the optimal products for the members would remain the same. Figure 10 and Table 6 summarize the results obtained. The table gives the values of $F$ for the least weight solution generated by having rigid connections at every beam-column junction. It also gives the values of $F$ for the minimal cost solution generated by minimizing Equation 5. As seen in Figure 10, the shape of the trade-off curve is dependent on the regional costs of material and labour. As can be perceived by intuition, for increasing values of $C_{r} / C_{s}$, significant cost savings can be achieved by minimizing the number of rigid connections. In the following sections, we present approaches to generate design alternatives.

\section{Generating design alternatives}

The solutions generated using optimization are not necessarily the best for adoption as a final design because such solutions are optimal only with respect to the modeled costs and constraints. Often, many objectives and constraints are not explicitly stated in the problem formulation due to their unquantifiable nature or difficulty in quantifying the relative importance of these objectives with respect to the modeled objectives. The solutions produced using an optimization approach may therefore be sub-optimal with respect to these unmodeled issues. Traditionally, this has been cited as a major limitation of optimization applications. Modeling to Generate Alternatives (MGA) techniques (Brill Jr. et al, 1990; Baugh Jr. et al, 1997) use optimization to generate a small set of very different solutions. These are an extension of single and multiple-objective mathematical 
programming techniques with an emphasis on generating a set of alternatives that are "good" but "as different as possible."

To illustrate MGA, consider the following formulation of a design optimization problem:

$$
\begin{aligned}
& \text { Maximize } z=x+2 y, \quad \text { subject to } x+y \leq 30 \\
& y \leq 20 \\
& x, y \geq 0
\end{aligned}
$$

The decision space for the problem is shown as the shaded region in Figure 11a. Mathematical optimization correctly produces $\mathrm{z}=50$ at point $\mathrm{A}$ as the solution. Thus, if the optimization model is an exact representation of all costs and objectives, the design would be $\langle x, y\rangle=\langle 10,20\rangle$. The premise of MGA, however, is that there may be features which are not completely captured by the model. When those issues are considered, point A may be less desirable overall than a set of other points $B$ originally deemed inferior by the model. The issues involved in finding $B$ and establishing the computer assistance needed in this process are discussed below.

- Generating all feasible solutions suggests that one has no confidence in the model. Thus, one would expect that all points in $B$ optimize the objective function nearly as well as A, so only good solutions need to be examined. This idea is illustrated in Figure 11b, where only those solutions that are above $90 \%$ of the optimal value are retained.

- Available solutions should represent a cross-section of good solutions, so that, if none of the solutions are actually in $B$, perhaps one of them is close enough from which to begin "tinkering."

- Since a decision maker can reasonably consider only a small number of designs, a subset of $B$ should be presented for further consideration.

For the problem defined above, the first alternative can be found by changing the formulation to maximize a difference metric $\delta$, instead of $z$ and imposing the constraint, $x+2 y \geq 0.9(50)$. The difference metric $\delta$ for this problem can be defined as $\delta^{2}=(x-10)^{2}+(y-20)^{2}$, which is the distance between the two points in the decision space. Similarly, MGA techniques can be applied for the design of steel moment frames if an appropriate difference metric can be devised.

\section{MGA for moment frame design}

We suggest using the "Hamming distance," which is a metric used in binary computation, as the difference metric for the MGA. If two solution strings are represented as $\left\langle c_{1 a}, c_{2 a}, . ., c_{R a}\right\rangle$ and $\left\langle c_{1 b}, c_{2 b}, \ldots, c_{R b}\right\rangle$, we can write,

$$
d_{a b}=\sum_{i=1}^{R}\left|c_{i a}-c_{i b}\right|
$$

where $d_{a b}$ is the difference between the two solutions. Let us consider that we have generated $n$ alternatives and are currently in the process of generating the $(n+1)$ th alternative. Then, we attempt to maximize the following difference metric to generate the $(n+1)$ th alternative.

$$
d=\sum_{j=1}^{n} d_{a j}
$$

Since the original model is accurate to a large extent, designers are interested in alternatives for which the costs are only slightly higher than the optimal cost. Therefore, we add the following constraint to the optimization formulation.

$$
F_{m g a} \leq k F_{\text {opt }}
$$


where $k>1$ and, $F_{m g a}$ and $F_{o p t}$ are the total equivalent weights of steel associated with the alternative and the optimal solution respectively.

The alternatives are generated by modifying Equation 10 to maximize $d$ instead of minimizing cost and adding an additional penalty to account for the constraint given by Equation 20. The value of $k$ is taken to be 1.1 , i.e., the search is for alternatives whose total costs are higher than the cost of the optimal solution by not more than $10 \%$. This study has not verified the robustness of this approach for identifying design alternatives. However, its performance is expected to be similar to the GA formulation used for the trade-off study and that has already been shown to be robust. The two GA formulations are the same except for a small difference in the objective function.

Using this formulation, we generate alternatives for $r_{r e q}=12$, when all the supports in the frame are fixed. These alternatives are evaluated on the basis of the following criteria - (1) preferences to certain locations for rigid connections, (2) margins against excessive lateral loads, and (3) number of W-shapes used in a single column of the frame.

The optimal cost solution for $r_{r e q}=12$ is given in Figure 12. The alternatives - MGA1 and MGA2, and their costs for $r_{r e q}=12$ are also shown in Figure 12. The empty circles on the beams represent locations of rigid connections. These two solutions along with the cost-optimal solution constitute a small solution set which the designer can explore with respect to the unmodeled objectives. All three solutions have significantly different locations for rigid connections. While the model assumes that all the rigid connections have the same cost, this is not entirely true in practice. The connection cost varies depending on the products used for the corresponding beam and column which in turn determine the required weld specifications, fabrication and stiffness requirements. In the optimal solution, the largest beam with a rigid connection uses W24 $\times 55$. In $M G A 1$, the largest beam with a rigid connection is member 19 with $\mathrm{W} 24 \times 68$. In $M G A 2$, the largest beam with a rigid connection is also member 19 but with $\mathrm{W} 24 \times 62$. Also, $M G A 1$ has most of its rigid connections on the innermost bay while $M G A 2$ and the optimal solution have the rigid connections primarily in the outermost bays. Since rigid connections require on-site welding, the engineer may consider construction aspects to determine if one alternative is superior to another. Specifically, engineers may prefer to have the rigid connections on the inner bays in a tight urban setting.

Another parameter that an engineer may consider to evaluate the relative quality of the alternatives is related to the ability of the frames to withstand increased lateral loads. While the generated solutions satisfy the strength constraint, it is possible that certain solutions have greater margins than others. An elegant way to compare the alternatives on this basis is by gradually increasing the wind load and identifying the wind load at which the different design alternatives fail. It is observed that the optimal solution fails when the wind load is increased by $95 \%$. On the other hand, the alternatives $M G A 1$ and $M G A 2$ fail when the wind load is increased by $123 \%$ and $117 \%$, respectively. The optimal solution, $M G A 1$, and $M G A 2$ have $F=46.26$, 50.80 and 46.68 metric tons respectively. Expertise and judgment can be used to evaluate the alternative that is best suited for final design.

The engineer may also wish to tinker with these solutions and arrive at a better solution. To illustrate this, let us consider the optimal solution and an alternative for the case when $r_{r e q}=6$ and the base of the frame is hinged. The respective solutions are shown in Figures 9 and 13. The solutions tend to have different products at each floor level for the same column. Engineers prefer to run the same column for atleast two floor levels as it reduces the the cost of splicing them. Therefore, the engineer can modify the solution so that the bottom two floors have the same $\mathrm{W}$-shape and the top three floors have the same $\mathrm{W}$-shape. The costs of the solutions are re-evaluated and the engineer may use discretion to identify the best among all the alternatives. In this manner, MGA and optimization can be combined to create a joint-cognitive decision-making environment, where the engineer uses computing tools to perform analyses, study trade-off curves and generate alternatives but employs engineering knowledge and experience to make the final decision.

\section{Summary and conclusions}

Computational approaches that can assist decision-makers in the design of rigid steel frames are proposed in this paper. Development of optimization-based approaches that enable designers to identify the best locations for rigid connections in a moment frame is presented. The optimization approach involves two components - 
(1) A simple iterative procedure to find the least weight solution when the frame is considered to have only rigid connections and (2) A Genetic Algorithm (GA) to perform trade-off study between the number of rigid connections and the total cost of the frame. The GA starts with the solution from the first algorithm and attempts to find the cost-optimal solutions as the number of rigid connections are gradually decreased. The GA uses a unique crossover scheme that enables it to find the least cost solution for a specified number of rigid connections by limiting the search to only those solutions in which the number of rigid connections is exactly equal to a specified number. The performance of this optimization technique is studied for a realistic 5-bay 5-story frame. The trade-off study is conducted for two support cases - (1) all the supports at the base are fixed and (2) all the supports at the base are hinged. Finally, MGA is applied to generate design alternatives since the solutions generated through optimization may not be optimal with respect to certain unmodeled objectives and constraints. The "Hamming distance" is used as the difference metric in the MGA to generate a handful of alternatives for moment frame design. These alternatives are compared with respect to the following criteria - (1) inherent margin in a particular design with respect to increased lateral loads, (2) preference of certain rigid connection locations in the frame based on construction aspects and (3) the number of product changes in a single column of the frame.

The following conclusions are derived from this study.

- The trade-off study shows that the total cost of the frame is optimal when the rigid connections are placed at only a few locations in the frame.

- When all the supports of the frame are fixed, there exists a trade-off between the number of rigid connections and the total cost.

- When all the supports in the frame are hinged, there is no clear trade-off between the number of rigid connections and the total cost. The total cost increases, in a general sense, with increasing numbers of rigid connections.

- MGA using "hamming distance" generates design alternatives that can prove valuable in arriving at a final design.

\section{References}

AISC (2001) Manual of Steel Construction - Load Resistance Factor Design, $3^{\text {rd }}$ edn. AISC

Al-Salloum YA, Almusallam TH (1995) Optimality and safety of rigidly- and flexibly-jointed steel frames. Journal of Constructional Steel Research 35:189-215

Balling RJ (1991) Optimal steel frame design by simulated annealing. Journal of Structural Engineering 117(6):1780-1795

Baugh Jr JW, Caldwell SC, Brill Jr ED (1997) A mathematical programming approach to generate alternatives in discrete structural optimization. Engineering Optimization 28:1-31

Brill Jr ED, Flach JM, Hopkins LD, Ranjithan S (1990) MGA: A decision support for complex, incompletely defined problems. IEEE Transactions on Systems, Man, and Cybernetics 20(4):745-757

Burns SA (ed) (2002) Recent Advances in Optimal Structural Design. ASCE

Chan CM, Wong K (2007) Structural topology and element sizing design optimization of tall steel frameworks using a hybrid oc-ga method. Structural and Multidisciplinary Optimization 35(5):473-488

Degertekin SO (2008) Optimum design of steel frames using harmony search algorithm. Structural and Multidisciplinary Optimization 36(4):393-401

Dhillon BS, O'Malley III JW (1999) Interactive analysis and design of flexibly connected frames. Journal of Structural Engineering 125(5):556-564 
Eiben AE, Smith JE (2003) Introduction to Evolutionary Computing. Springer

Foley CM, Schinler D (2003) Automated design of steel frames using advanced analysis and object-oriented evolutionary computation. Journal of Structural Engineering 129(5):648-660

Foley CM, Vinnakota S (1999a) Inelastic behavior of multistory partially restrained steel frames, Part I. Journal of Structural Engineering 125(8):854-861

Foley CM, Vinnakota S (1999b) Inelastic behavior of multistory partially restrained steel frames, Part II. Journal of Structural Engineering 125(8):862-869

Fragiadakis M, Lagaros ND, Papadrakakis M (2006) Performance-based multiobjective optimum design of steel structures considering life-cycle cost. Structural and Multidisciplinary Optimization 32:1-11

Goldberg DE (1989) Genetic algorithms in Search, Optimization and Machine Learning. Addison Wesley Publishing Company

Gupta A, Kripakaran P, Kumar G, Baugh Jr JW (2005) Genetic Algorithm-based decision support for optimizing seismic response of piping systems. Journal of Structural Engineering 131(3):389-398

Haupt RL, Haupt SE (2004) Practical Genetic Algorithms. John Wiley \& sons

Hayalioglu MS, Degertekin SO (2004) Design of non-linear steel frames for stress and displacement constraints with semi-rigid connections via genetic optimization. Structural and Multidisciplinary Optimization 27(4):259-271

Hayalioglu MS, Degertekin SO (2005) Minimum cost design of steel frames with semi-rigid connections and column bases via genetic optimization. Computers and Structures 83:1849-1863

Kameshki ES, Saka MP (2001) Optimum design of nonlinear steel frames with semi-rigid connections using a genetic algorithm. Computers and Structures 79(17):1593-1604

Kameshki ES, Saka MP (2007) Optimum geometry design of nonlinear braced domes using genetic algorithm. Computers and Structures 85:71-79

Kargahi M, Anderson JC, Dessouky MM (2006) Structural weight optimization of frames using tabu search, I: Optimization procedure. Journal of Structural Engineering 132(12):1858-1868

Kicinger R, Arciszewski T, DeJong K (2005) Evolutionary design of steel structures in tall buildings. Journal of Computing in Civil Engineering 19(3):223-238

Kripakaran P, Gupta A (2006) MGA - a mathematical approach to generate alternatives. In: Smith IFC (ed) Intelligent Computing in Engineering and Architecture, Springer, Lecture Notes in Computer Science, pp $408-415$

Liu M, Burns SA, K WY (2006) Genetic algorithm based construction-conscious minimum weight design of seismic steel moment-resisting frames. Journal of Structural Engineering 132(1):50-58

Loughlin DH, Neal JK, Ranjithan S, Brill Jr ED, Baugh Jr JW (1995) Decision support system for air quality management. In: Computing in Civil Engineering: Proceedings of the 2nd Congress, ASCE, pp 1367-1374

Machaly ESB (1986) Optimum weight analysis of steel frames with semi-rigid connections. Computers and Structures 23(4):565-574

Michalewicz Z, Fogel DB (2004) How to Solve It: Modern Heuristics. Springer

Mitchell M (1998) An Introduction to Genetic Algorithms. The MIT Press

Patnaik SN, Hopkins DA (1998) Optimality of a fully-stressed design. Computer Methods in Applied Mechanics and Engineering 165(1-4):215-221 
Rajeev S, Krishnamoorthy CS (1997) Genetic algorithms-based methodologies for design optimization of trusses. Journal of Structural Engineering 123(3):350-358

Saka MP (2007) Optimum design of steel frames using stochastic search techniques based on natural phenomena: A review. In: Topping BHV (ed) Civil Engineering Computations: Tools and Techniques, SaxeCoburg Publications

Simoes LMC (1996) Optimization of frames with semi-rigid connections. Computers and Structures 60(4):531-539

Xu L, Grierson DE (1993) Computer-automated design of semirigid steel frameworks. Journal of Structural Engineering 119(6):1740-1760

Xu L, Grierson DE (2006) Seismic design optimization of steel building frameworks. Journal of Structural Engineering 132(2):277-286 
Table 1: Concentrated loads for frame given in Figure 1

\begin{tabular}{ccc}
\hline \hline Load type & Joints & Load $(\mathrm{kN})$ \\
\hline \multirow{2}{*}{$W$} & 1,6 & 32.11 \\
& $2,3,4,5$ & 64.18 \\
\hline & 6,36 & 6.67 \\
$D$ & 12,30 & 26.68 \\
& 18,24 & 50.44 \\
& $2,3,4,5,32,33,34,35$ & 44.92 \\
& $8,9,10,11,26,27,28,29$ & 64.05 \\
$L$ & $14,15,16,17,20,21,22,23$ & 195.26 \\
\hline \multirow{3}{*}{$L$} & $2,3,4,5,32,33,34,35$ & 26.69 \\
& $8,9,10,11,26,27,28,29$ & 106.75 \\
$L_{r}$ & $14,15,16,17,20,21,22,23$ & 202.38 \\
\hline & 6,36 & 17.08 \\
& 12,30 & 68.32 \\
& 18,24 & 129.53 \\
\hline
\end{tabular}

Table 2: Vertical distributed loads (y-direction) for frame given in Figure 1

\begin{tabular}{ccc}
\hline \hline $\begin{array}{c}\text { Load } \\
\text { type }\end{array}$ & Members & $\begin{array}{c}\text { Load } \\
(\mathrm{kN} / \mathrm{m})\end{array}$ \\
\hline \multirow{3}{*}{$D$} & $7-10,17-20,37-40,47-50$ & 16.05 \\
& $27-30$ & 7.88 \\
& $6,16,36,46$ & 4.38 \\
& 26 & 1.17 \\
\hline \multirow{2}{*}{$L$} & $7-10,17-20,37-40,47-50$ & 17.51 \\
& $27-30$ & 4.38 \\
\hline$L_{r}$ & $6,16,26,36,46$ & 2.77 \\
\hline
\end{tabular}

Table 3: Product set (W-shapes) for the beams in frame shown in Figure 1

\begin{tabular}{cccc}
\hline \hline $10 \times 12$ & $16 \times 40$ & $24 \times 68$ & $33 \times 130$ \\
$12 \times 14$ & $18 \times 35$ & $24 \times 76$ & $36 \times 135$ \\
$12 \times 16$ & $18 \times 40$ & $24 \times 84$ & $36 \times 194$ \\
$12 \times 19$ & $21 \times 44$ & $27 \times 84$ & $40 \times 149$ \\
$12 \times 22$ & $21 \times 48$ & $30 \times 90$ & $40 \times 167$ \\
$12 \times 26$ & $21 \times 50$ & $30 \times 99$ & $40 \times 183$ \\
$14 \times 22$ & $21 \times 55$ & $30 \times 108$ & $40 \times 199$ \\
$14 \times 26$ & $24 \times 55$ & $30 \times 116$ & $40 \times 211$ \\
$16 \times 26$ & $24 \times 62$ & $33 \times 118$ & $40 \times 215$ \\
$16 \times 31$ & & & \\
\hline
\end{tabular}


Table 4: Product set (W-shapes) for the columns in frame shown in Figure 1

\begin{tabular}{cccc}
\hline \hline $14 \times 22$ & $14 \times 74$ & $14 \times 176$ & $14 \times 398$ \\
$14 \times 26$ & $14 \times 82$ & $14 \times 193$ & $14 \times 426$ \\
$14 \times 30$ & $14 \times 90$ & $14 \times 211$ & $14 \times 455$ \\
$14 \times 34$ & $14 \times 99$ & $14 \times 233$ & $14 \times 500$ \\
$14 \times 38$ & $14 \times 109$ & $14 \times 257$ & $14 \times 550$ \\
$14 \times 43$ & $14 \times 120$ & $14 \times 283$ & $14 \times 605$ \\
$14 \times 48$ & $14 \times 132$ & $14 \times 311$ & $14 \times 665$ \\
$14 \times 53$ & $14 \times 145$ & $14 \times 342$ & $14 \times 730$ \\
$14 \times 61$ & $14 \times 159$ & $14 \times 370$ & $14 \times 808$ \\
$14 \times 68$ & & & \\
\hline
\end{tabular}

Table 5: Least weight solution for fully rigid frame shown in Figure 1

\begin{tabular}{cc}
\hline \hline W-shape & Members \\
\hline \multirow{2}{*}{$14 \times 120$} & $1,2,11,12,21,22$ \\
& $31,32,41,42,51,52$ \\
\hline \multirow{3}{*}{$14 \times 53$} & $3,4,5,13,14,15,23$ \\
& $24,25,33,34,35,43$ \\
& $44,45,53,54,55$ \\
\hline \multirow{2}{*}{$18 \times 35$} & $8,9,10,18,19,20$ \\
& $28,29,30,38,39$ \\
& $40,48,49,50$ \\
\hline $12 \times 14$ & $6,16,36,46$ \\
\hline $14 \times 22$ & $7,17,26,37,47$ \\
\hline $16 \times 26$ & 27 \\
\hline
\end{tabular}

Table 6: Optimal cost for different values of $C_{r} / C_{s}$ when base is fixed for 5-bay 5-story frame

\begin{tabular}{ccccc}
\hline \hline Cost Ratio & \multirow{2}{*}{$\begin{array}{c}\text { F for } \\
\text { least-weight }\end{array}$} & \multicolumn{2}{c}{$\begin{array}{c}\text { Optimal cost } \\
\text { solution }\end{array}$} & \multirow{2}{*}{$\begin{array}{c}\text { reduction } \\
\text { in } F\end{array}$} \\
\cline { 3 - 4 }$(\mathrm{CR})$ & design (tons) & $r_{\text {req }}$ & $F$ (tons) & \\
\hline 1.00 & 65.50 & 12 & 40.83 & $38 \%$ \\
1.25 & 76.82 & 12 & 43.55 & $43 \%$ \\
1.50 & 88.16 & 10 & 46.00 & $48 \%$ \\
1.75 & 99.50 & 10 & 48.26 & $51 \%$ \\
2.00 & 112.66 & 10 & 50.53 & $55 \%$ \\
\hline
\end{tabular}




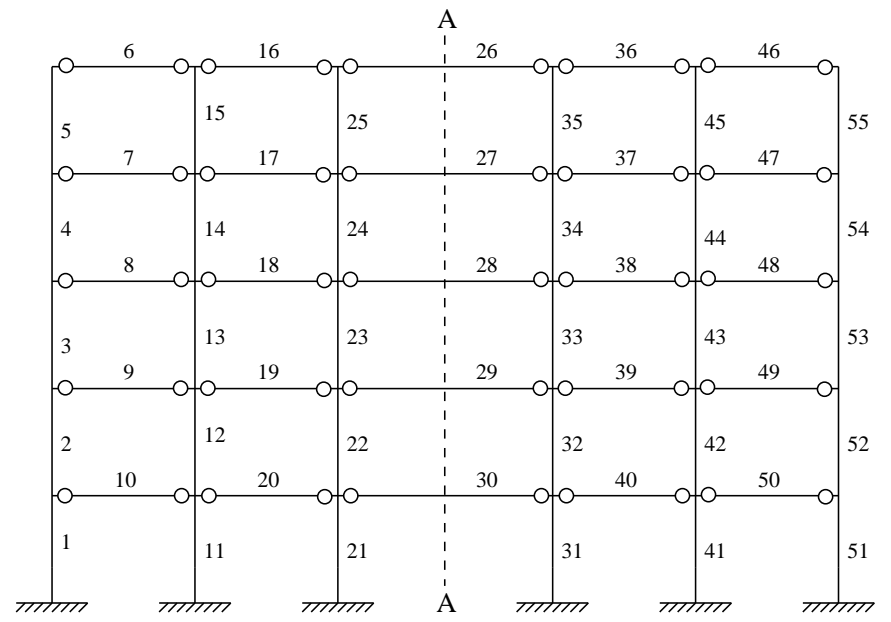

Figure 1: Elevation of 5-bay 5-story frame with element numbers beside the corresponding elements. Possible locations for rigid connections are indicated using a circle beside the respective beam-column junctions

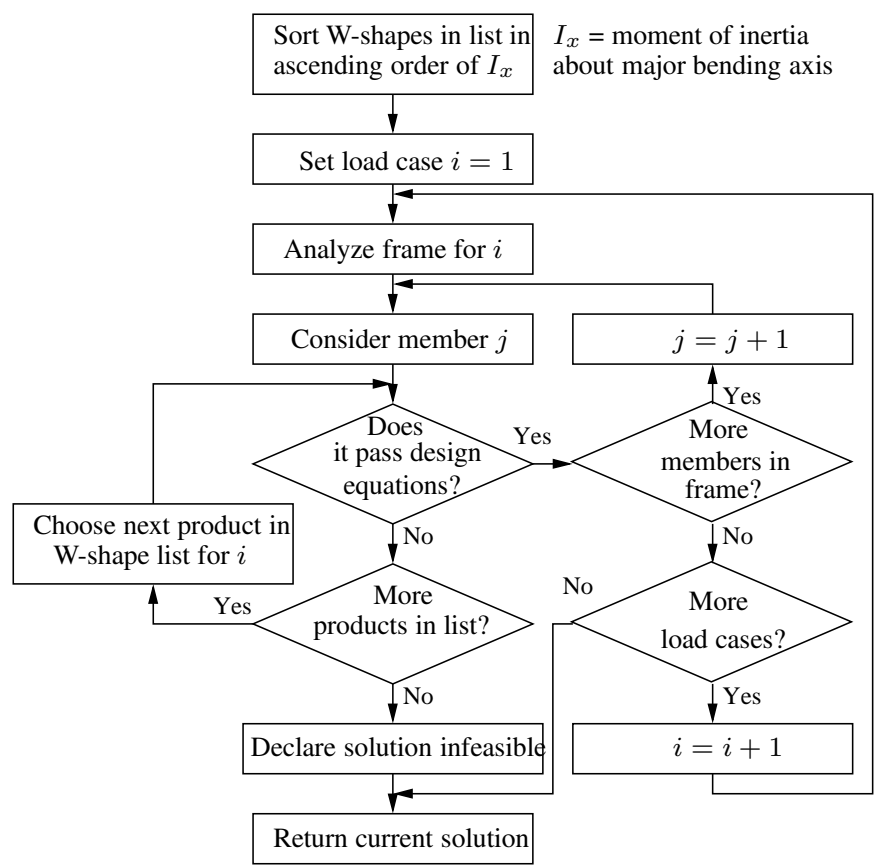

Figure 2: Algorithm for correcting solutions that violate equation 6 and 7 


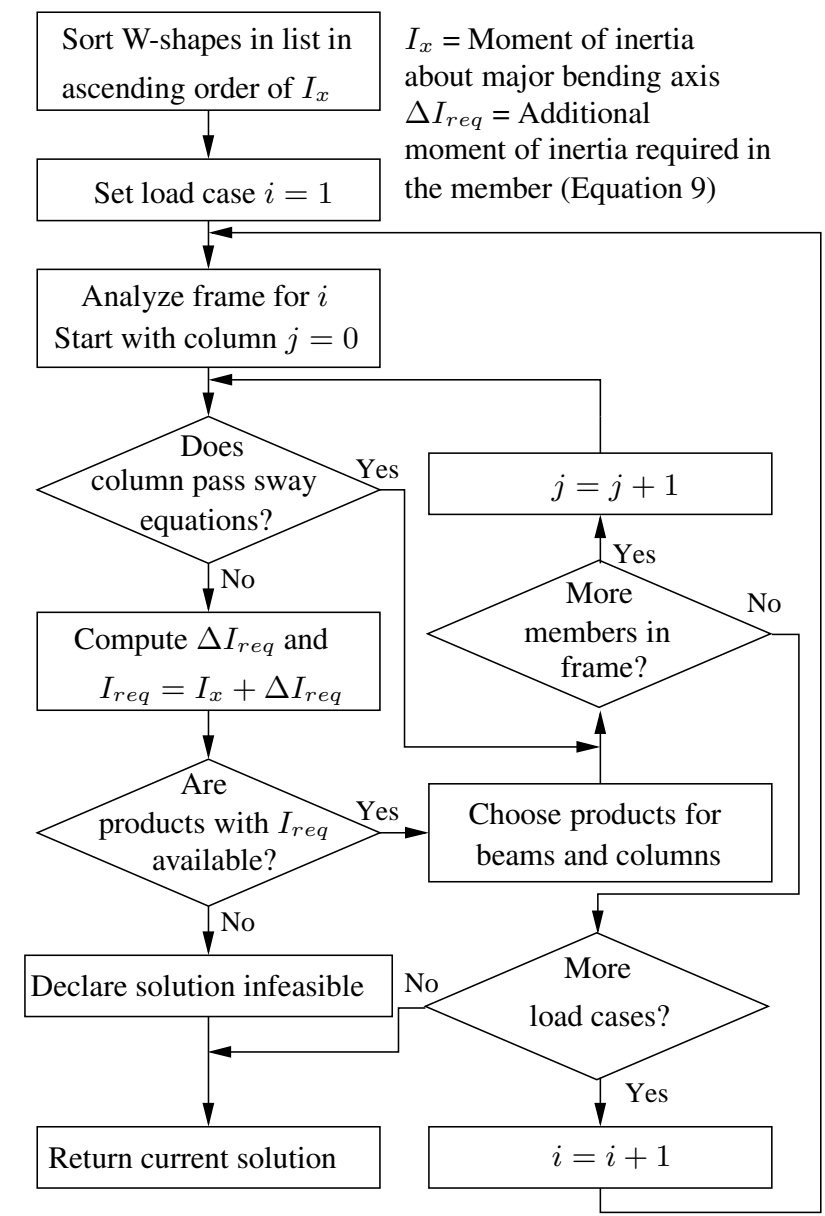

Figure 3: Algorithm for correcting solutions that violate sway constraints

\begin{tabular}{|c|c|c|c|c|c|}
\hline 1 & 2 & 3 & 4 & 5 & \\
\hline 1 & 1 & 0 & & & \\
\hline & 0 & $Q$ & & & \\
\hline
\end{tabular}

\begin{tabular}{ll|l|l|l|l|l|}
\hline & 0 & 0 & 0 & 0 & 1 & Offspring 1 \\
Y & 0 & 0
\end{tabular}

$S=\{2,6,7,8\} ; S_{1}=\{2,8\} ; S_{2}=\{6,7\}$

Figure 4: Illustration of the crossover operator 


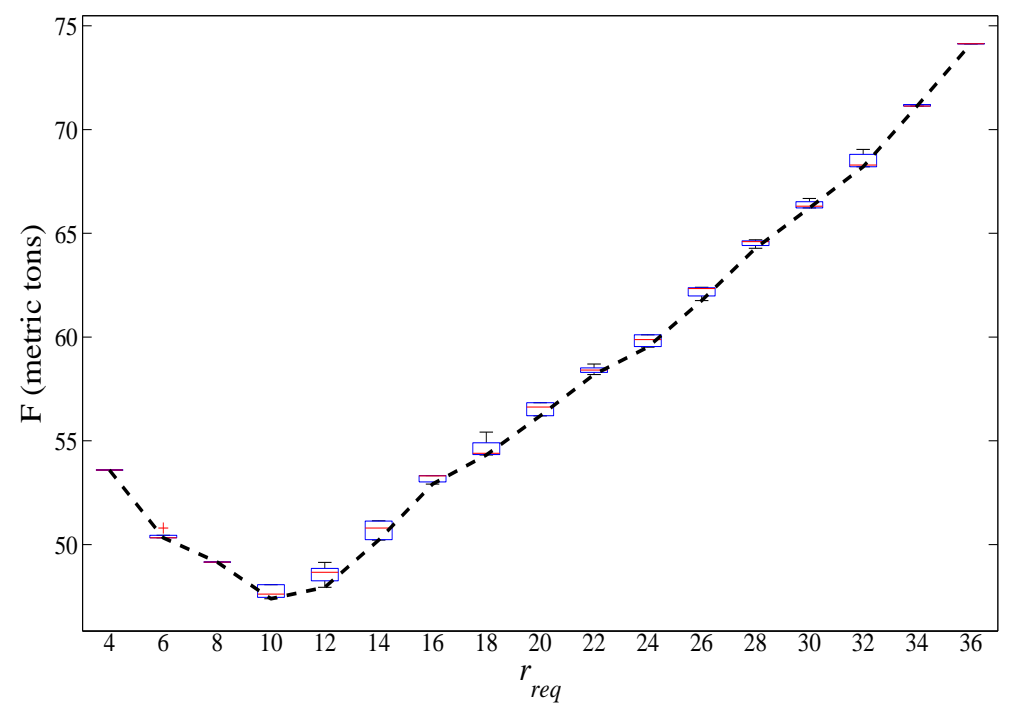

Figure 5: Box-plot showing the range of cost values obtained when minimizing the total cost of the frame shown in Figure 1 with a fully rigid base for various values for $r_{r e q}$

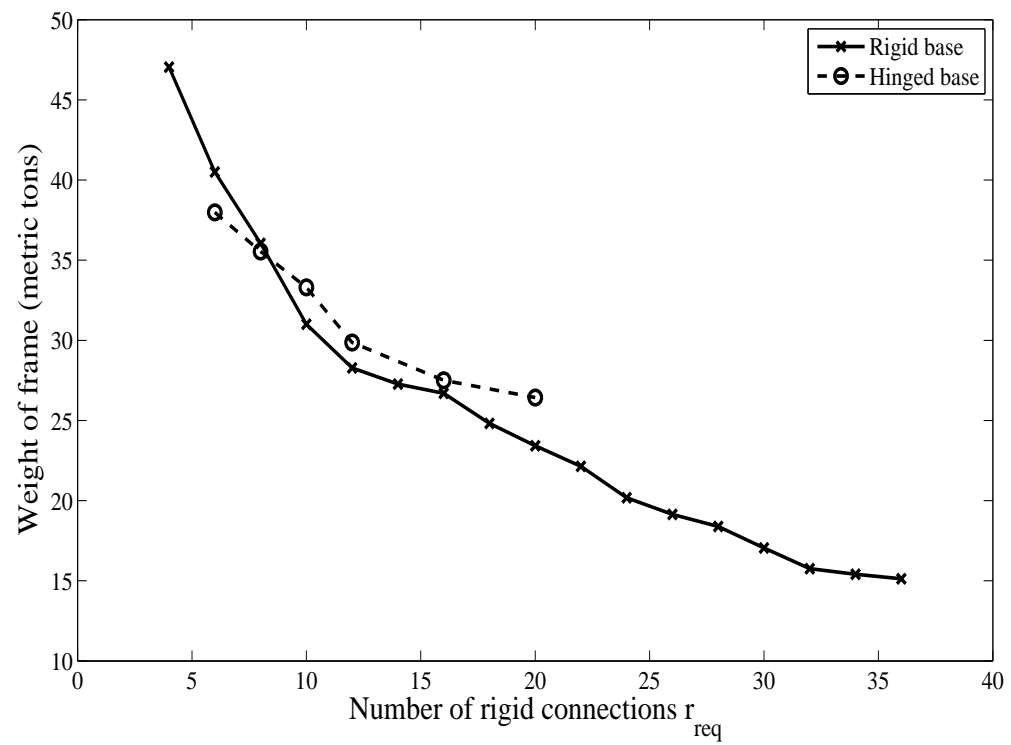

Figure 6: Plot of the trade-off between material cost and number of rigid connections for the frame shown in Figure 1 


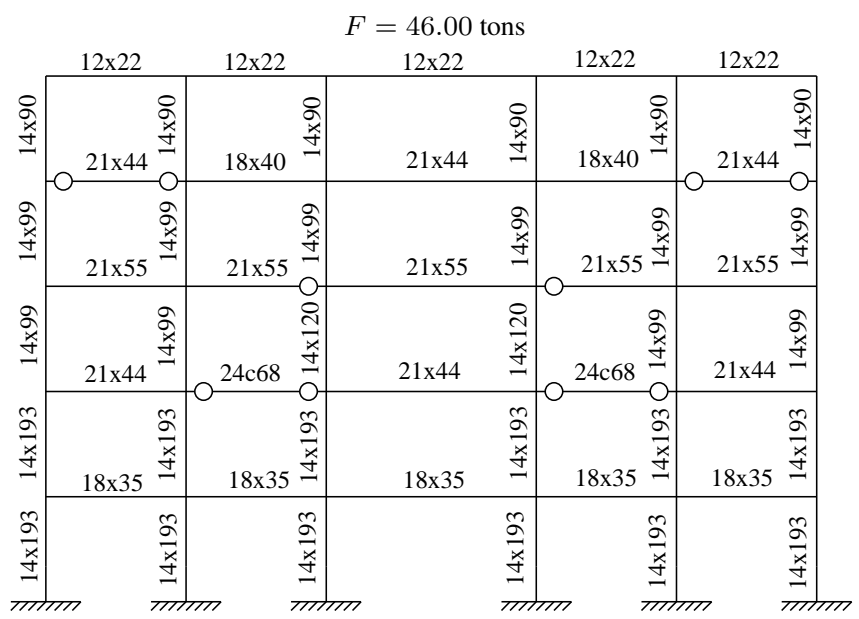

Figure 7: Rigid connection locations and W-shapes in cost optimal solution for 5-bay 5-story frame with fixed base

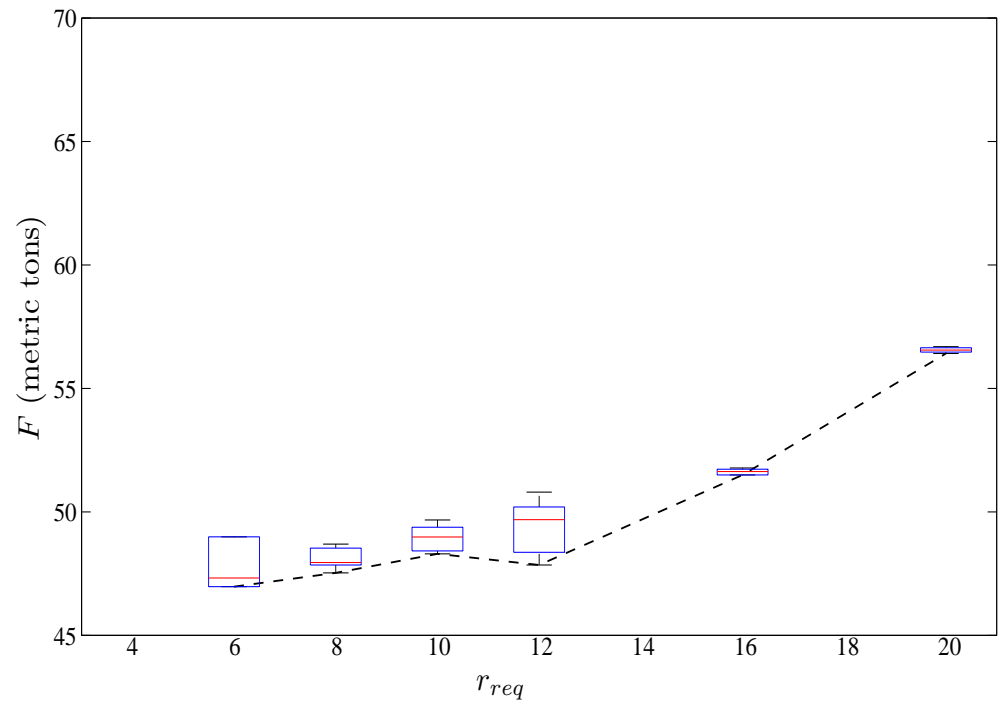

Figure 8: Box-plot showing the range of cost values obtained when minimizing the total cost of the frame shown in Figure 1 with a fully hinged base for various values for $r_{r e q}$ 


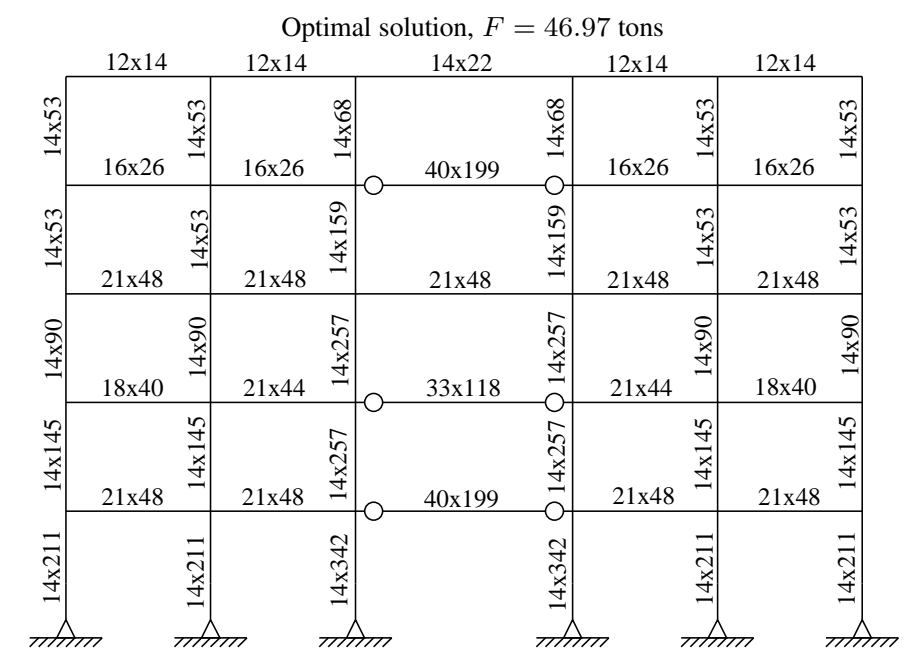

Figure 9: Rigid connection locations and W-shapes in cost optimal solution for 5-bay 5-story frame with hinged base

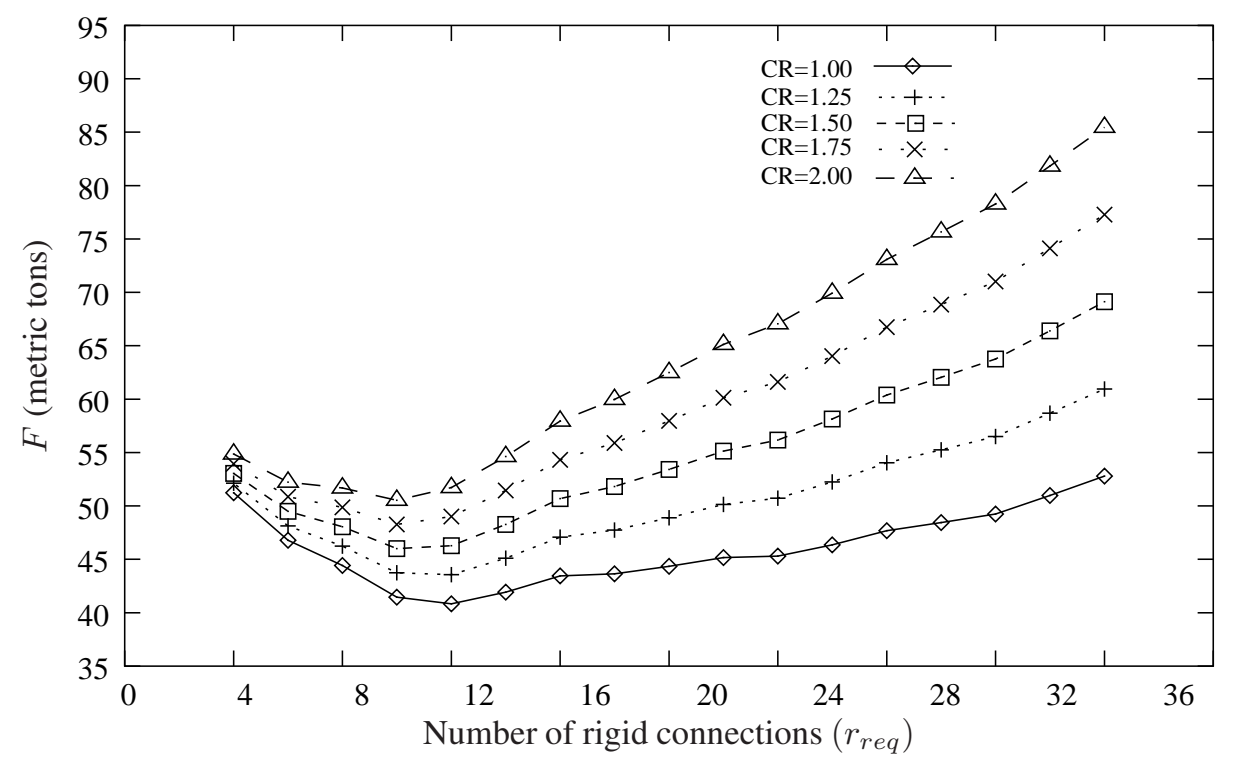

Figure 10: Plot showing trade-off curve for different values of $\mathrm{CR}=C_{r} / C_{s}$ 


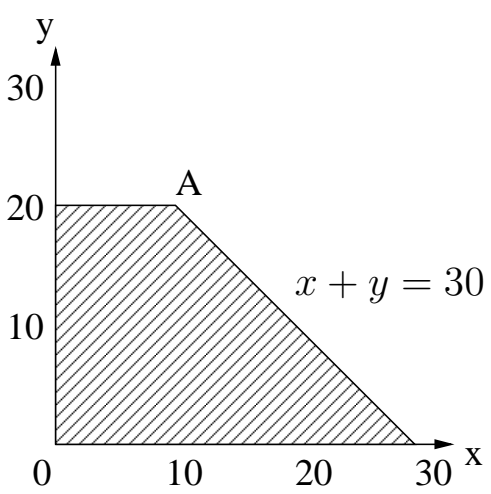

(a) Decision space

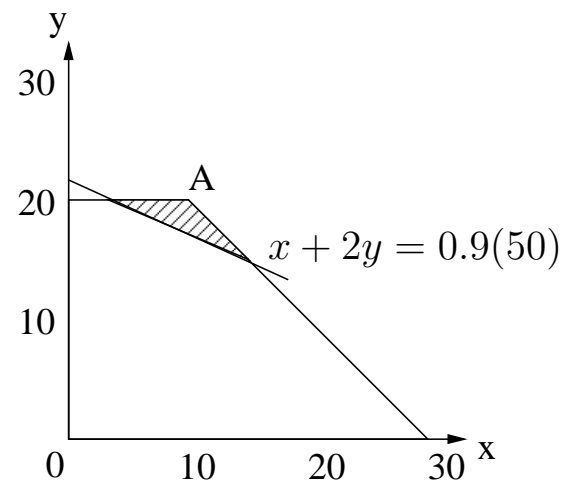

(b) Reduced decision space

Figure 11: Illustration of MGA 
Optimal solution, $F=46.26$ tons

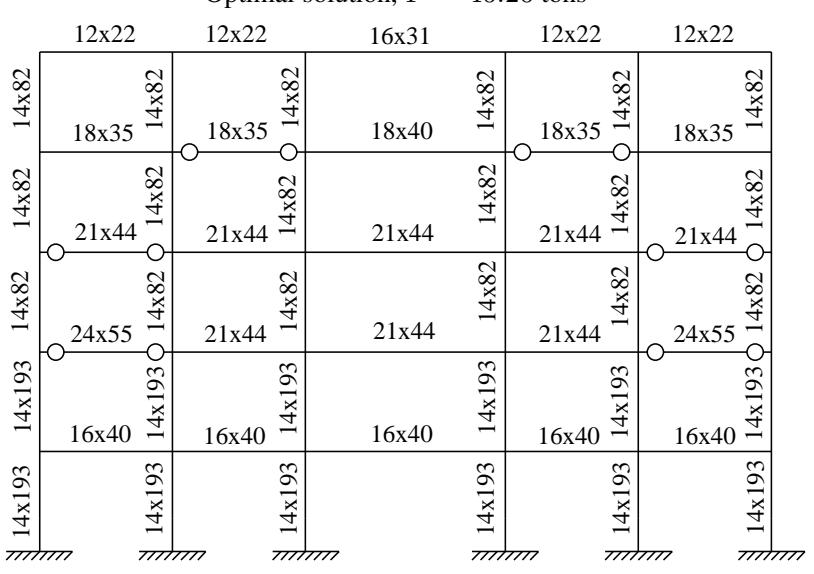

$M G A 1, F=50.80$ tons

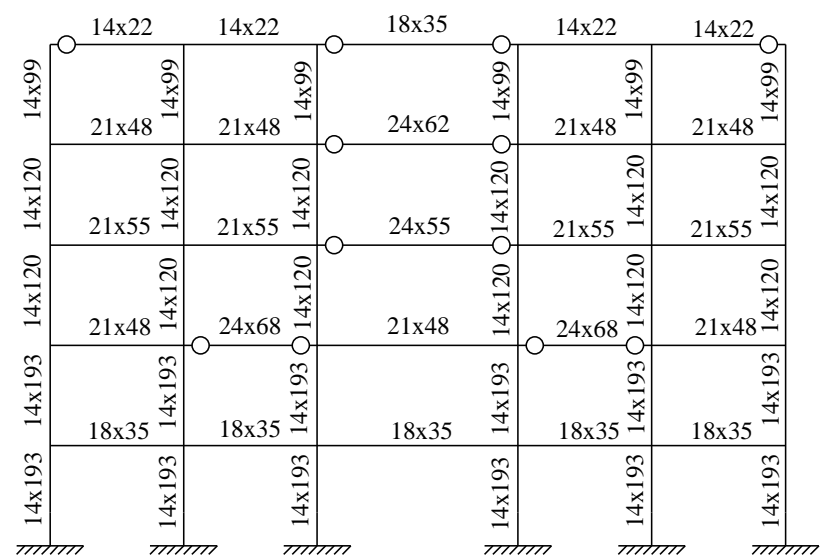

$M G A 2, F=46.68$ tons

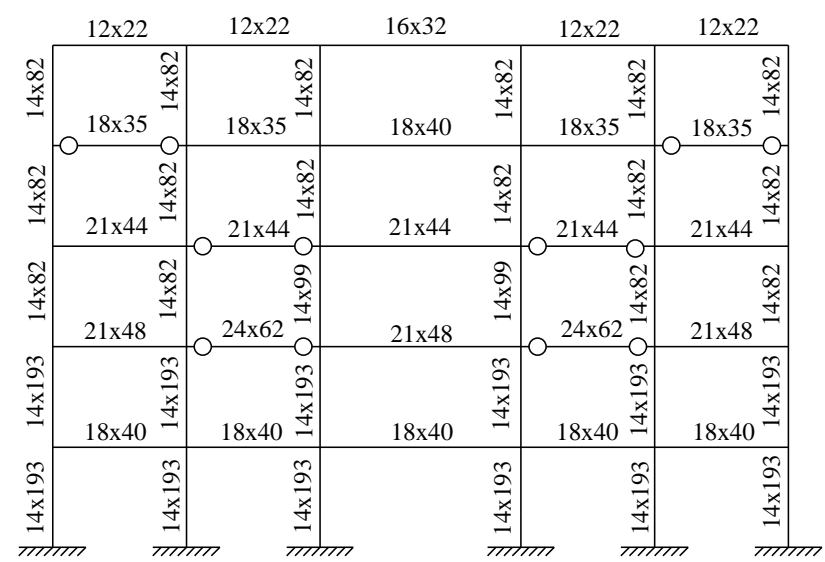

Figure 12: Rigid connection locations and W-shapes in optimal cost solution and alternatives when all supports are fixed and $r_{r e q}=12$ 

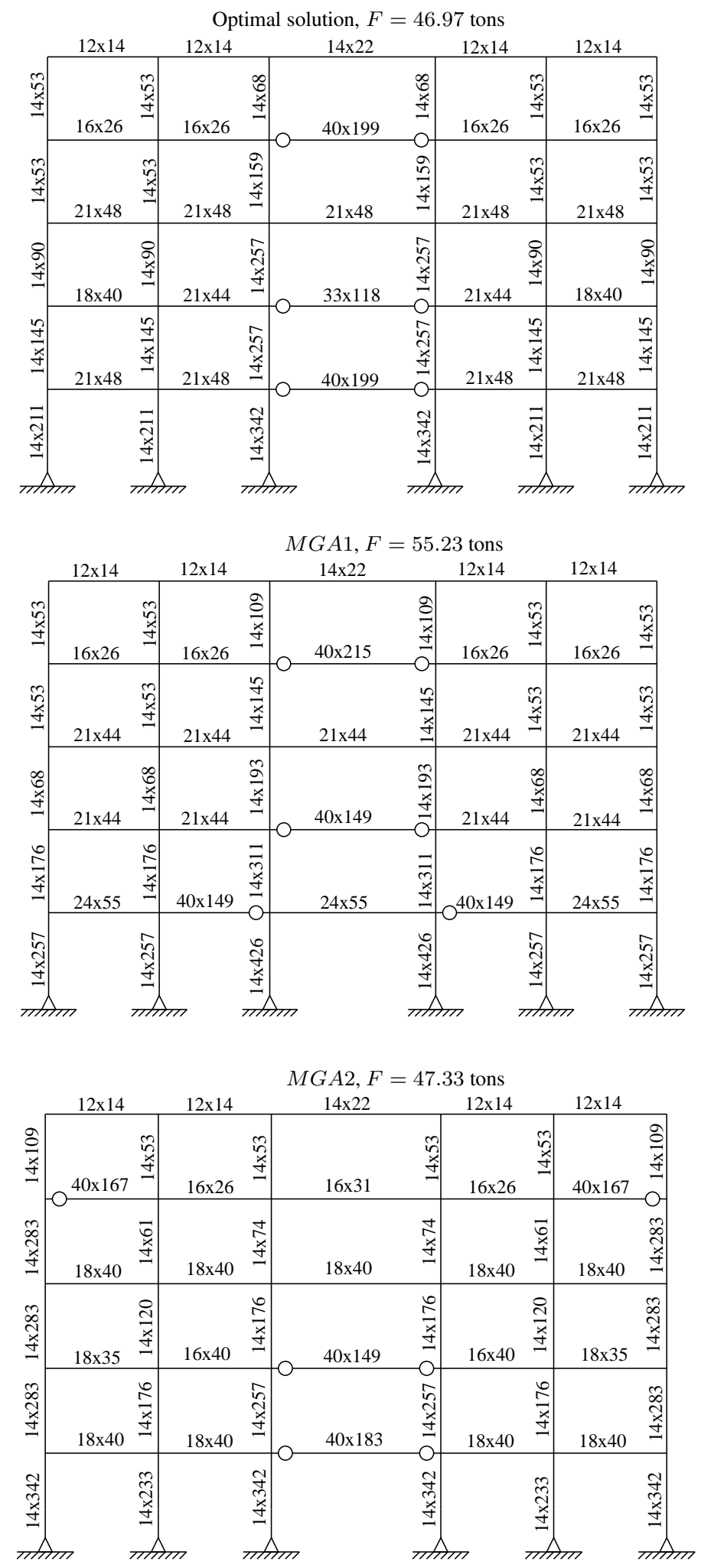

Figure 13: Rigid connection locations and W-shapes in $M G A 1$ when all supports are hinged and $r_{r e q}=6$ 